\title{
Wolfram syndrome: Portuguese research
}

\author{
Cristina Ferreras ${ }^{1,2}$, Vanessa Gorito ${ }^{1,2}$, Jorge Pedro ${ }^{3}$, Sofia Ferreira ${ }^{2,4}$, Carla Costa ${ }^{2,4}$, Rita Santos Silva ${ }^{2,4}$, \\ Cintia Castro Correia ${ }^{2,4}$
}

${ }^{1}$ Department of Paediatrics, Centro Hospitalar São João, Porto, Portugal

${ }^{2}$ Faculty of Medicine, University of Porto, Porto, Portugal

${ }^{3}$ Department of Endocrinology, Centro Hospitalar São João, Porto, Portugal

${ }^{4}$ Pediatric Endocrinology and Diabetology Unit, Department of Paediatrics, Centro Hospitalar São João, Porto, Portugal

\begin{abstract}
Introduction: Wolfram syndrome (WFS) is a neurological and endocrinological degenerative disorder, also known as DIDMOAD (Diabetes Insipidus, early-onset Diabetes Mellitus, progressive Optic Atrophy, and Deafness) syndrome. It is an autosomal recessive disorder, mostly involving the Wolfram syndrome 1 gene (WFS1). The phenotypic pleiomorphism, rarity, and molecular complexity complicate the follow-up of these patients.

Material and methods: We aimed to describe the clinical characteristics and the follow-up of 11 patients with this disorder. We retrospectively analysed all WFS patients diagnosed between 1990 and 2020 in the Centro Hospitalar São João, a tertiary hospital in Northern Portugal. Results: Eleven patients were included. Four patients had all 4 components of DIDMOAD. The presentation was diabetes mellitus (DM) in 9 patients, optic atrophy (OA) in another patient, and diabetes insipidus (DI) in another one. The median age of DM and OA diagnosis was 6 and 14 years, respectively. Nine patients had diabetes mellitus, and the other 2 patients had impaired glucose tolerance. All patients had OA. Four patients presented DI, all of them diagnosed in adolescence. Four patients had hearing impairment, 5 had urological abnormalities, 5 had neurological disorders, and 8 had psychiatry disorders. Eight patients had a broad spectrum of recessive mutations in WFS1. Conclusion: The information obtained in this study can facilitate further research in an attempt to improve prevention strategies for this devastating disease. (Endokrynol Pol 2021; 72 (4): 353-356)
\end{abstract}

Key words: Wolfram syndrome; diabetes mellitus; diabetes insipidus; optic atrophy; deafness

\section{Introduction}

Wolfram syndrome (WFS), also known as DIDMOAD, is a rare inherited disease first described in 1938 by Wolfram and Wagener [1, 2]. It is characterized by diabetes insipidus (DI), diabetes mellitus (DM), optic atrophy (OA), and deafness. Urological, psychological, and neurological comorbidities may also be present.

Wolfram syndrome is very rare. Its prevalence has been estimated at 1 in 770,000 individuals in the UK, 1 in 500,000 in Germany, 1 in 710,000 in the Japanese population, and 1 in 68,000 in the Lebanese population. The highest prevalence, of 1 in 54,478, is in a small district in a Sicilian population [2-6]. The high prevalence of WFS in Lebanese and Sicilian populations could be due to the high rates of consanguinity in these populations $[7,8]$.

Because WFS is a progressive disorder, and affected individuals experience a wide spectrum of symptoms during their lifetimes. The lifespans of affected individuals are generally shortened as a consequence of neurological and psychiatric problems, such as central respiratory failure, food aspiration, and suicide. Currently, no therapeutic intervention is known to alter the progression or the life expectancy of the affected individuals [9].

Several loss-of-function mutations of the Wolfram syndrome 1 gene (WFS1) have been described in patients with WFS [4]. WFS1-deficient mice have glucose intolerance associated with loss of pancreatic beta cells. The gene product of WFS1 is an endoplasmic reticulum embedded protein, which has been implicated in various cellular functions such as insulin secretion and processing, cell cycle regulation, unfolded protein response, and cAMP production [10-15]. On the other hand, WFS1 mutation was not identified in some patients, providing evidence of genetic heterogeneity for this disease. A specific mutation in a second gene (WFS2), also known as CISD2, has been described in affected Jordanian families [16].

The natural history of WFS has been characterized in different populations, mainly Asian populations, and there are few singles in occidental European populations. 


\section{Material and methods}

We retrospectively analysed all WFS patients diagnosed between 1990 and 2020 in the Centro Hospitalar São João, a tertiary hospital in Northern Portugal.

We screened a total of 14 patients who were eligible for our study due to the fact that they had diagnostic clinical criteria and identification of WFS1 mutations. One patient clinically suspected of WFS was excluded because he had no genetic confirmation, and 2 patients with clinical and genetic confirmation were excluded because they had no follow-up in our hospital in adult life.

Demographic data, clinical presentation, including signs and symptoms, and their onset age, family history, and genetic analysis were retrospectively abstracted from clinical records.

Descriptive statistics were expressed for quantitative data by the median. They were presented for categorical data as the number and percentage.

\section{Results}

Our population comprised 11 WFS patients, with 7 females $(63 \%)$ and a median current age of 23 years (range 12-34 years). Two patients were siblings. The patients' clinical features are summarized in Table 1. Four patients $(36.3 \%)$ had all 4 components of DIDMOAD. OA was present in all patients. Diabetes mellitus was present in $9(81.8 \%)$ patients, and the other 2 patients had impaired glucose tolerance (IGT) rather than DM. Eight patients $(72.7 \%)$ presented with psychiatric disorders. Urological abnormalities were present in 5 of the 11 patients $(45.4 \%)$. Five patients also had neurological disorders. Four patients (36.4\%) had insipidus diabetes, and 4 patients (36.4\%) also had hearing impairment. One patient presented a rheumatological disorder: undifferentiated arthritis in need of biological treatment.

The first clinical manifestation of the disease occurred at a median age of 7 years (range 2-15 years), all of them in paediatric age, and consisted of diabetes mellitus in 9 patients $(81 \%), \mathrm{OA}$ in another patient, and DI in another one. The median age of DM, OA, DI, and urological disorders at onset was 6 (range 2-11 years), 14 (range 5-30 years), 13 (range 10-16 years), and 10 (range 8-10 years), respectively. Hearing loss (HL) developed at the median age of 8 years (range 6-15 years).

Nine patients have diabetes and are currently treated with insulin, in a median dose of 0.7 units $/ \mathrm{kg} /$ day. The current median $\mathrm{HbA}_{1 \mathrm{c}}$ is $7.5 \%$ (range 7-9.5\%). Mild hypoglycaemia was found in all patients, and non-proliferative retinopathy was found in 1 patient who had had diabetes for 20 years.

Optic atrophy was bilateral and progressive in all patients, and presented as hypoplasia, visual field defect, and colour blindness - all of them diagnosed in paediatric age.

Three patients presented DI, all of them diagnosed in adolescence. Urological abnormalities including residual urine in the bladder and urinary incontinence were established by imaging examinations in $5(45.4 \%)$ of the 11 patients.

All patients had mutations in WFS1. Eight patients $(72.7 \%)$ had a broad spectrum of recessive mutations in WFS1. Three patients (27.2\%) had mutations in only 1 allele. Ages at onset of DM in patients with recessive WFS1 mutations were lower than those in patients with 1 mutation in only 1 allele.

Two patients had changes in brain MRI at this time, which showed atrophy of the cerebellum and brain stem, 1 of them with recent hospitalization in intensive care for acute respiratory failure. The overall survival rate was $100 \%$ at the time of follow-up, and the age of patients ranged from 12 to 34 years.

Table 1. Demographic features of the patients and detailed clinical presentations

\begin{tabular}{|c|c|c|c|c|c|c|c|c|c|}
\hline Case $\mathbf{n}^{0}$ & Onset age [yrs] & Sex & DM/IGT & $\mathbf{O A}$ & DI & HL & UD & ND & PD \\
\hline 1 & 8 & $\mathrm{M}$ & Yes & Yes & Yes & Yes & Yes & Yes & Yes \\
\hline 2 & 3 & $\mathrm{~F}$ & Yes & Yes & Yes & Yes & Yes & Yes & Yes \\
\hline 3 & 3 & $\mathrm{~F}$ & Yes & Yes & No & No & Yes & No & Yes \\
\hline 4 & 6 & $\mathrm{M}$ & Yes & Yes & No & No & No & No & Yes \\
\hline 5 & 7 & $\mathrm{M}$ & Yes & Yes & No & No & No & No & Yes \\
\hline 6 & 7 & $\mathrm{~F}$ & Yes & Yes & No & No & No & Yes & Yes \\
\hline 7 & 6 & $M$ & Yes & Yes & No & No & No & No & No \\
\hline 8 & 14 & $\mathrm{~F}$ & Yes & Yes & Yes & Yes & Yes & No & Yes \\
\hline 9 & 15 & $\mathrm{~F}$ & Yes & Yes & No & No & No & No & No \\
\hline 10 & 11 & $\mathrm{~F}$ & Yes & Yes & Yes & Yes & No & Yes & Yes \\
\hline 11 & 6 & $\mathrm{~F}$ & Yes & Yes & No & No & Yes & Yes & No \\
\hline
\end{tabular}

$\mathrm{M}$ - male; F — female; DM — diabetes mellitus; IGT — impaired glucose tolerance; $\mathrm{OA}$ — optic atrophy; DI — diabetes insipidus; HL — hearing loss;

UD — urological disorder; ND — neurological disorder; PD — psychological disorder 


\section{Discussion}

In this study, we retrospectively described the clinical features of 11 WFS patients from a single centre in the last 30 years.

Diabetes mellitus was the most common clinical feature in our cohort, in accordance with previous studies. Non-auto-immune insulin-dependent DM is the first manifestation of WFS, but there is a great diagnostic delay from the onset of diabetes to the diagnosis of the syndrome. Zmyslowska et al. found that WFS1 was diagnosed with a delay of at least 7 years, and that all WFS1 patients were primarily misdiagnosed as having insulin-dependent type 1 DM [17]. This could be a limitation of our study.

Microvascular complications are rare, and they do not progress as quickly as in the type 1 DM. WFS1 non-autoimmune insulin-dependent DM is characterized by a daily insulin requirement and a mean $\mathrm{HbA}_{1 \mathrm{c}}$ lower than in type $1 \mathrm{DM}$, because in WFS1 the residual insulin secretion lasts longer than in type 1 DM. In our sample, we verified the same tendency. Non-autoimmune insulin-dependent DM of WFS begins in preschool-age without ketoacidosis, it is antibody-negative, and has surprisingly long periods of remission. Therefore, WFS must be suspected in these cases [18-20].

Optic atrophy is usually present in the diagnosis of WFS. In these patients, OA occurs in the first decade; it is progressive and often leads to blindness. It begins at an average age of 11 years ( 6 weeks to 19 years) with reduced visual acuity and loss of colour vision $[2,18]$. The median age of diagnosis of OA in our patients was 14 years. Hence, in WFS patients, an annual eye examination is essential, including visual acuity, colour vision testing, fundoscopy, visual field, and optical coherence tomography scan. The monitoring of the efficacy of potential therapy can be performed by visual evoked potentials [6].

In WFS patients, diabetes mellitus usually occurs in the first decade of life, OA is diagnosed during the early second decade, DI and HL during the second decade, and urological and neurological abnormalities during $10-30$ years [21-23], as in our study.

Generally sensorineural deafness presents at an average age of $12-16$ years (range $5-39$ years) and is a feature seen in $62 \%$ of WFS patients [2,9]. This disorder affects high frequencies first and progresses relatively slowly [2]. Pennings et al. found that in WFS females, hearing loss was more prevalent than in WFSF1 males [24]. However, in other studies, no gender differences in the degree of deafness were found [2,25]. In our study, we found that 2 females and 1 male presented this disorder. The follow-up of this disorder in WFS patients includes an audiometry test every year or every 2 years [6].

Neurological complications and psychiatric disorders are frequent in WFS patients. In our study, the incidence of neurological complications was $45 \%$. The most common symptom is cerebellar ataxia, as in our study. Headache has also been reported in WFS [4] - 5 patients in our study presented headache. These patients should be evaluated yearly or twice a year by a neurologist.

Urinary tract problems are another major clinical challenge for Wolfram syndrome patients, affecting $60-90 \%$ of this population [26]. Hydroureteronephrosis, urinary incontinence, and recurrent infections are common signs of neurogenic bladder. The median age of onset of urological manifestations is 20 years, although numerous patients develop the symptoms at 10-20 years of age $[6,28]$. We reported urological abnormalities in $45.4 \%$ of patients.

Frequently (up to 20-30\%) WFSF patients are affected by episodes of severe depression, psychosis, smell and sleep abnormalities, or organic brain symptoms, as well as impulsive verbal and physical aggression, while WFS1 heterozygotes may be predisposed to psychiatric illness. Usually, cognitive and psychiatric symptoms begin in the later stages of the disease [4, 26-28]. In our case, we found a high number of patients with this pathology, given that up $72 \%$ of our patients presented psychiatric disorders from adolescence onwards.

Genetic analysis should be considered as an effective method to assist diagnosis and genetic consultation [6]

Wolfram syndrome has high morbidity and mortality, without effective treatment, and the median age of death is around 30 years (range 25-49). Respiratory failure or dysphagia due to brainstem involvement are common causes of mortality $[6,29]$. In our case, thus far the survival rate is $100 \%$, with the age of patients ranging from 12 to 34 years.

More studies are necessary to better manage this devastating disease and to guarantee the patients a better quality of life and longer life expectancy [30].

\section{Conclusions}

The information obtained in this study can facilitate further research in an attempt to improve prevention strategies, as well as treatments for this devastating disease.

In the author's opinion, Wolfram syndrome, as a multisystemic progressive disease requiring rapid diagnosis, should be followed up in tertiary hospital centres, by a multidisciplinary team. Special care should be taken in the transition into adulthood follow-up, taking into account that the greatest worsening of the disease occurs from the third decade of life. 


\section{References}

1. Marquardt J. Diabetes Mellitus and Optic Atrophy. Arch Int Med. 1974; 134(1): 32, doi: 10.1001/archinte.1974.00320190034003.

2. Barrett TG, Bundey SE, Macleod AF. Neurodegeneration and diabetes: UK nationwide study of Wolfram (DIDMOAD) syndrome. Lancet. 1995; 346(8988): 1458-1463, doi: 10.1016/s0140-6736(95)92473-6, indexed in Pubmed: 7490992.

3. Minton JAL, Rainbow LA, Ricketts C, et al. Wolfram syndrome. Rev Endocr Metab Disord. 2003; 4(1): 53-59, doi: 10.1023/a:1021875403463, indexed in Pubmed: 12618560.

4. de Heredia ML, Clèries R, Nunes V. Genotypic classification of patients with Wolfram syndrome: insights into the natural history of the disease and correlation with phenotype. Genet Med. 2013; 15(7): 497-506, doi: 10.1038/gim.2012.180, indexed in Pubmed: 23429432

5. Rohayem J, Ehlers C, Wiedemann B, et al. Wolfram Syndrome Diabetes Writing Group. Diabetes and neurodegeneration in Wolfram syndrome: a multicenter study of phenotype and genotype. Diabetes Care. 2011; 34(7): 1503-1510, doi: 10.2337/dc10-1937, indexed in Pubmed: 21602428.

6. Rigoli L, Lombardo F, Salzano G, et al. Wolfram syndrome and WFS1 gene. Clin Genet. 2011; 79(2): 103-117, doi: 10.1111/j.1399-0004.2010.01 522.x, indexed in Pubmed: 20738327.

7. Medlej R, Wasson J, Baz P, et al. Diabetes mellitus and optic atrophy: a study of Wolfram syndrome in the Lebanese population. J Clin Endocrinol Metab. 2004; 89(4): 1656-1661, doi: 10.1210/jc.2002-030015, indexed in Pubmed: 15070927.

8. Zalloua PA, Azar ST, Delépine M, et al. WFS1 mutations are frequent monogenic causes of juvenile-onset diabetes mellitus in Lebanon. Hum Mol Genet. 2008; 17(24): 4012-4021, doi: 10.1093/hmg/ddn304, indexed in Pubmed: 18806274.

9. Kumar S. Wolfram syndrome: important implications for pediatricians and pediatric endocrinologists. Pediatr Diabetes. 2010; 11(1): 28-37, doi: 10.1111/j.1399-5448.2009.00518.x, indexed in Pubmed: 20015125.

10. Riggs AC, Bernal-Mizrachi E, Ohsugi M, et al. Mice conditionally lacking the Wolfram gene in pancreatic islet beta cells exhibit diabetes as a result of enhanced endoplasmic reticulum stress and apoptosis. Diabetologia. 2005; 48(11): 2313-2321, doi: 10.1007/s00125-005-1947-4, indexed in Pubmed: 16215705 .

11. Akiyama M, Hatanaka M, Ohta $Y$, et al. Increased insulin demand promotes while pioglitazone prevents pancreatic beta cell apoptosis in Wfs1 knockout mice. Diabetologia. 2009; 52(4): 653-663, doi: 10.1007/s00125-009-1270-6, indexed in Pubmed: 19190890.

12. Hatanaka M, Tanabe K, Yanai A, et al. Wolfram syndrome 1 gene (WFS1) product localizes to secretory granules and determines granule acidification in pancreatic beta-cells. Hum Mol Genet. 2011; 20(7): 1274-1284, doi: 10.1093/hmg/ddq568, indexed in Pubmed: 21199859.

13. Yamada T, Ishihara H, Tamura A, et al. WFS1-deficiency increases endoplasmic reticulum stress, impairs cell cycle progression and triggers the apoptotic pathway specifically in pancreatic beta-cells. Hum Mol Genet. 2006; 15(10): 1600-1609, doi: 10.1093/hmg/ddl081, indexed in Pubmed: 16571599.

14. Gharanei S, Zatyka M, Astuti D, et al. Vacuolar-type H+-ATPase V1A subunit is a molecular partner of Wolfram syndrome 1 (WFS1) protein, which regulates its expression and stability. Hum Mol Genet. 2013; 22(2): 203-217, doi: 10.1093/hmg/dds400, indexed in Pubmed: 23035048

15. Wang DD, Hu FY, Gao FJ, et al. The Precise Diagnosis of Wolfram Syndrome Type 1 Based on Next-Generation Sequencing. Front Genet. 2019; 10: 1217, doi: 10.3389/fgene.2019.01217, indexed in Pubmed: 31850070.
16. Amr S, Heisey C, Zhang M, et al. A homozygous mutation in a novel zinc-finger protein, ERIS, is responsible for Wolfram syndrome 2 . Am J Hum Genet. 2007; 81(4): 673-683, doi: 10.1086/520961, indexed in Pubmed: 17846994.

17. Zmyslowska A, Borowiec M, Fichna P, et al. Delayed recognition of Wolfram syndrome frequently misdiagnosed as type 1 diabetes with early chronic complications. Exp Clin Endocrinol Diabetes. 2014; 122(1): 35-38, doi: 10.1055/s-0033-1357160, indexed in Pubmed: 24464595.

18. Rigoli L, Lombardo F, Salzano G, et al. Identification of one novel causative mutation in exon 4 of WFS1 gene in two Italian siblings with classical DIDMOAD syndrome phenotype. Gene. 2013; 526(2): 487-489, doi: 10.1016/j.gene.2012.10.023, indexed in Pubmed: 23103830.

19. Ganie MA, Bhat D. Current developments in Wolfram syndrome. J Pediatr Endocrinol Metab. 2009; 22(1): 3-10, doi: 10.1515/jpem.2009.22.1.3, indexed in Pubmed: 19344068.

20. Cano A, Molines L, Valéro R, et al. French Group of Wolfram Syndrome. Microvascular diabetes complications in Wolfram syndrome (diabetes insipidus, diabetes mellitus, optic atrophy, and deafness [DIDMOAD]): an age- and duration-matched comparison with common type 1 diabetes. Diabetes Care. 2007; 30(9): 2327-2330, doi: 10.2337/dc07-0380, indexed in Pubmed: 17536072.

21. Zmysłowska A, Borowiec M, Fendler W, et al. The prevalence of Wolfram syndrome in a paediatric population with diabetes. Endokrynol Pol. 2014; 4: 65, doi: 10.5603/EP.2014.0040, indexed in Pubmed: 25185852.

22. Duan L, Li Q, Tong AL, et al. Clinical Characteristics of Wolfram Syndrome in Chinese Population and a Novel Frameshift Mutation in . Front Endocrinol (Lausanne). 2018; 9: 18, doi: 10.3389/fendo.2018.00018, indexed in Pubmed: 29483894.

23. Matsunaga K, Tanabe K, Inoue H, et al. Wolfram syndrome in the Japanese population; molecular analysis of WFS1 gene and characterization of clinical features. PLoS One. 2014; 9(9): e106906, doi: 10.1371/journal. pone.0106906, indexed in Pubmed: 25211237.

24. Pennings RJE, Huygen PLM, van den Ouweland JMW, et al. Sex-related hearing impairment in Wolfram syndrome patients identified by inactivating WFS1 mutations. Audiol Neurootol. 2004; 9(1): 51-62, doi: 10.1159/000074187, indexed in Pubmed: 14676474.

25. Cremers CW, Wijdeveld PG, Pinckers AJ. Juvenile diabetes mellitus, optic atrophy, hearing loss, diabetes insipidus, atonia of the urinary tract and bladder, and other abnormalities (Wolfram syndrome). A review of 88 cases from the literature with personal observations on 3 new patients. Acta Paediatr Scand Suppl. 1977(264): 1-16, doi: 10.1111/j.1651-2227.1977.tb15069.x, indexed in Pubmed: 270276.

26. Soares A, Mota Á, Fonseca S, et al. Ophthalmologic Manifestations of Wolfram Syndrome: Report of 14 Cases. Ophthalmologica. 2019; 241(2): 116-119, doi: 10.1159/000490535, indexed in Pubmed: 30056456.

27. Hasan MA, Hazza I, Najada A. Wolfram's (DIDMOAD) Syndrome and Chronic Renal Failure. Saudi J Kidney Dis Transpl. 2000; 11(1): 53-58, indexed in Pubmed: 18209300.

28. Chaussenot A, Bannwarth S, Rouzier C, et al. Neurologic features and genotype-phenotype correlation in Wolfram syndrome. Ann Neurol. 2011; 69(3): 501-508, doi: 10.1002/ana.22160, indexed in Pubmed: 21446023.

29. Urano F. Wolfram Syndrome: Diagnosis, Management, and Treatment. Curr Diab Rep. 2016; 16(1): 6, doi: 10.1007/s11892-015-0702-6, indexed in Pubmed: 26742931.

30. Pallotta MT, Tascini G, Crispoldi R, et al. Wolfram syndrome, a rare neurodegenerative disease: from pathogenesis to future treatment perspectives. J Transl Med. 2019; 17(1): 238, doi: 10.1186/s12967-019-1993-1, indexed in Pubmed: 31337416. 